www.jmscr.igmpublication.org

Impact Factor 5.84

Index Copernicus Value: 71.58

ISSN (e)-2347-176x ISSN (p) 2455-0450

crossref DOI: _https://dx.doi.org/10.18535/jmscr/v5i9.13

\title{
Management of unstable inter-trochanteric fractures by Dynamic Hip screw versus Proximal Femur Nailing- A Comparative Analysis
}

\author{
Authors \\ Dr Surendar Singh Bava, Dr Tushar Kadam \\ SETH GSMC \& KEMH
}

\begin{abstract}
Background: The aim of this study was to compare the outcomes of proximal femoral nail (PFN) and dynamic hip screw (DHS) in treatment of unstable intertrochanteric fractures.

Material and Methods: In our study, we included 80 unstable intertrochanteric fractures, out of which 40 were treated with PFN and 40 were treated with DHS. The surgeries were performed on traction table and were followed up at regular intervals at 2 weeks, 4 weeks, 6 months and annually thereafter.

Results: The functional results were evaluated with Harris Hip Score. At most recent follow up, Patients with excellent results were $23(46 \%)$ in group A and 20 (36\%) in group B while patients with good results were 15(28\%) in group A and 20(45\%) in group B. Differences were observed to be statistically significant difference between two groups in view of late \& early complications and time to union. Better outcomes were noted in PFN group for unstable intertrochanteric fractures.

Conclusions: We concluded that PFN may be the better fixation device for most unstable intertrochanteric fractures.
\end{abstract}

\section{Introduction}

Per-trochanteric fractures include those occurring in the region from the extra-capsular basilar neck region to the region along the lesser trochanter proximal to the medullary canal. Intertrochanteric and peritrochanteric fractures are generic terms for pertrochanteric fractures. The injury creates a spectrum of fractures in the proximal metaphyseal region of the bone, with damage to the intersecting cancellous compression and tensile lamellar networks and the weak cortical bone. This results in displacement of fracture fragments and attached muscle groups. This region of the femur shares many common biomechanical properties with other metaphyseal-diaphyseal fractures with regard to difficulty in obtaining stable fixation. Though predominantly occurring with low-energy trauma in older patients, high energy trauma in younger patients can cause similar fracture patterns.

The intertrochanteric fracture pattern constitutes one of the most commonly operated fracture types globally, with high post-operative fatality rates and have high cost of care required after surgery. Gullberg et al. in 1997 estimated the future incidence of hip fractures worldwide. They estimated that the incidence would double to 2.6 million by the year 2025 and 4.5 million by the year 2050 .

The classification of intertrochateric fractures have not been particularly helpful in clinical situations. However, increased surgical complexity and recovery is associated with unstable fracture patterns. The unstable characteristics include 
posteromedial fragmentation, basicervical pattern, reverse oblique pattern, displaced greater trochanteric/lateral wall fractures, and failure to reduce the fracture prior to internal fixation. Stability after surgical treatment implies union without deformity or implant failure. The multiple classification schemes used for inter-trochanteric fractures include
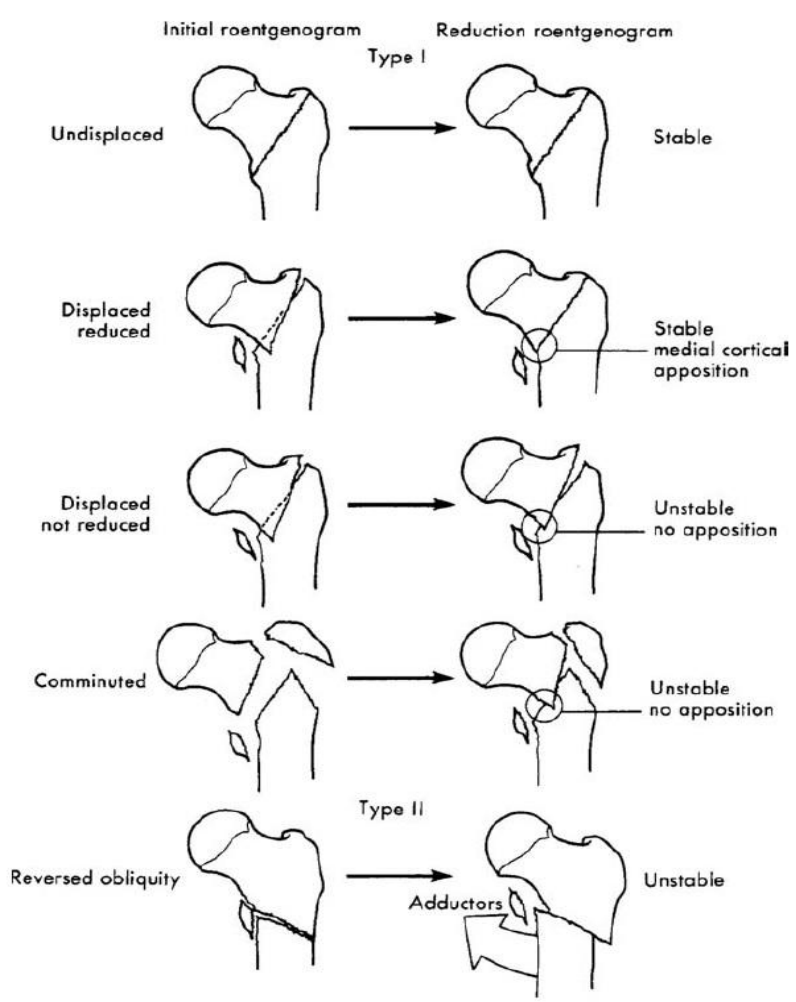

Evans' classification of intertrochanteric fractures.

(Reproduced with permission from Bucholz, R. W. \& Heckman, J. D. Rockwood and Green's Fractures in Adults, vol. 2. Philadelphia: Lippincott Williams and Wilkins, 1991.)

\section{Evan's Classification}

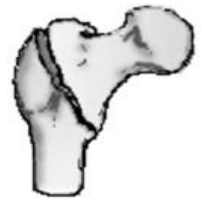

31-A1.1

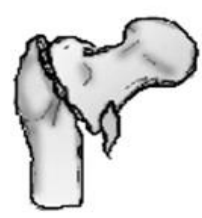

31-A2.1

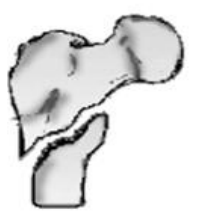

31-A3.1

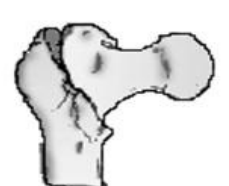

31-A1.2

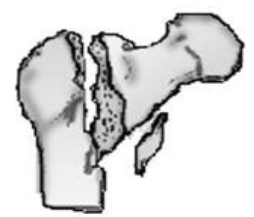

31-A2.2

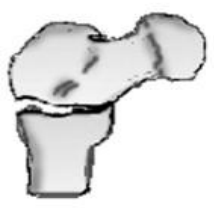

31-A3.2

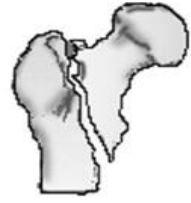

31-A1.3

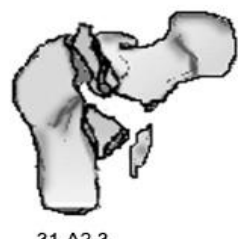

31-A2.3

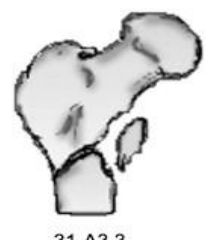

31-A3.3
OTA alphanumeric classification

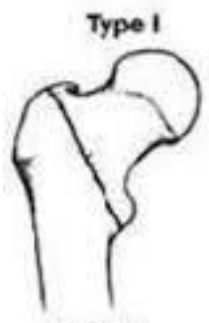

Type II
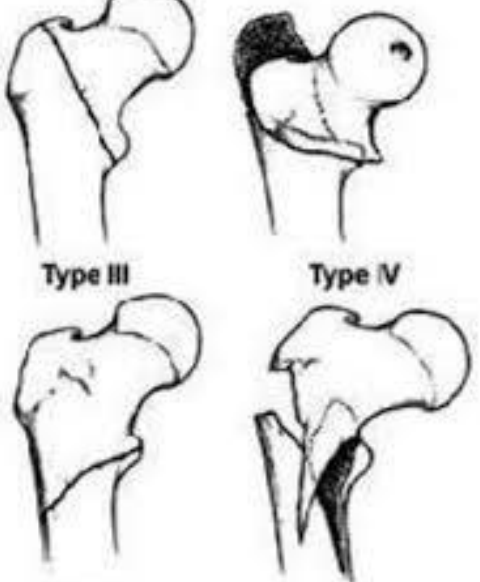

Boyd-Griffin classification
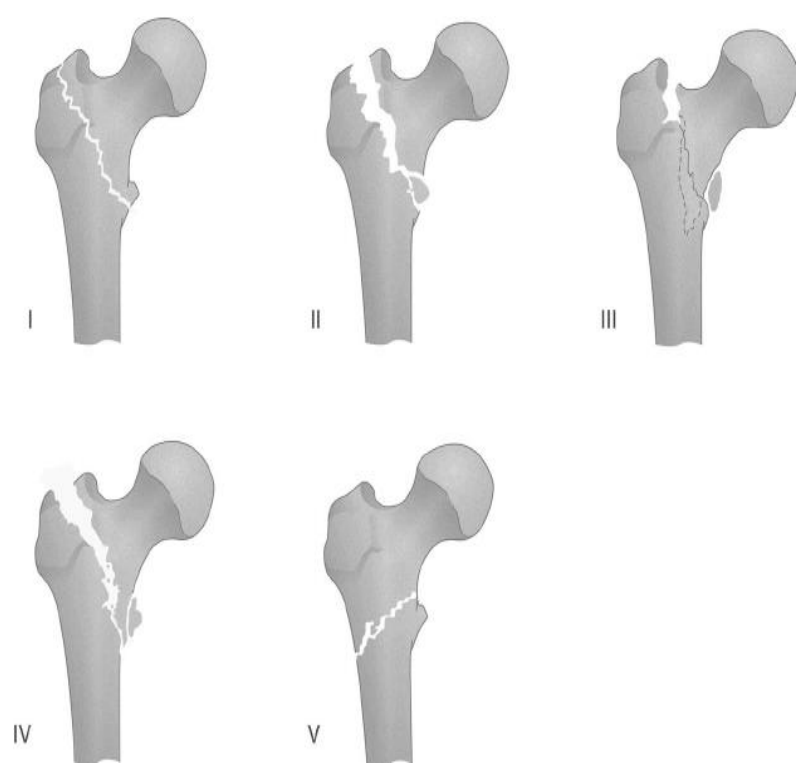

Tronzo classification

Nonoperative treatment of intertrochanteric fractures is rare but may still have a role in nonambulatory patients in whom adequate pain control can be achieved without surgery. Internal fixation is appropriate for most inter-trochanteric fractures. Optimal fixation is based on the stability of the fracture. The mainstay of treatment is fixation with a screw-side plate device or an intramedullary nail.

Unstable IT fractures can be managed by either Dynamic Hip Screw (DHS) or Proximal Femoral Nailing (PFN). DHS has gained widespread acceptance and has produced good results but complications are frequent, particularly in unstable inter-trochanteric fracture. The advantage 
of PFN is that it provides a more biomechanically stable construct by reducing the distance between hip joint and implant. While DHS is a load bearing implant, PFN is a load sharing one. Each implant offers certain advantages over the other.

Our study aimed to compare the outcomes of fixation of unstable inter-trochanteric fractures by DHS versus PFN. The objectives of this study were - 1) To compare methods of fixation of unstable IT fracture of femur in adults with respect to intra operative parameters 2) Toevaluatethe functional outcome with respect to return to function, and complications in the two groups. 3)To ascertain which implant would be better suited for this fracture type so as to provide the best results with the least complications. 4)To evaluate the operated cases over a long-term for any residual loss of function and overall tolerability of implant.

\section{Materials and Methods}

Study was based as a double blind randomized control study. It was conducted on the patients with unstable IT fractures admitted in the orthopaedics department after obtaining the required consent subject toethical clearance was obtained from the institutional ethics committee. The study period was 2 years from June 2014 to June 2016. The study was conducted among the study population after obtaining written informed consent. Our study population consisted of 80 patients (40 in each group). Out of 80 cases, 40 were treated by proximal femoral nailing (PFNgroup A) and 40 were treated by dynamic hip screw (DHS-group B). The groups were allocated randomly. AO/OTA classification for per trochanteric fractures was used.

According to AO/OTA classification -

31A1 - fractures are simple, two-part fractures,

31A2 - fractures have multiple fragments

$31 \mathrm{~A} 3$ - fractures includes reverse oblique and transverse fracture patterns

2.1 Inclusion criteria 1) Patients who were in the age group of more than 45 years of either sex 2) Unstable Intertrochanteric fracture type 31-A 1/2/3 (OTA classification)
2.2 Exclusion criteria 1) Patients with medical comorbidities who were declared unfit for the surgery 2) pathological fractures 3) Polytrauma patients 4) Pre-existing hip/femoral deformity 6) Sub-trochanteric fractures

The relevant information was collected. Initial radio-graph of the pelvis with both hip joints was done. The 80 patients were divided in to two groups, 40 in eac, randomnly. The patients under group A were treated by PFN and patients under group B were treated by DHS. Their was an average delay of surgery in our study of 7 days (mean 2-10 days). All surgeries were performedon the traction table following closed reduction, confirmed with image intensifier guidance on two different views.

For PFN,a standard length PFN (250 mm) was used in all our cases. The diameter of the femur wass measured at the level of isthmus on an AP $\mathrm{X}$-ray and Neck shaft angle was measured in unaffected side in AP X-ray.

For DHS, neck shaft angle was determined using X-ray AP view on unaffected side and length of side plate was determined to allow purchase of at least 8 cortices on the shaft, distal to the fracture. Post-operatively, all patients were startedon physiotherapy in addition to early mobilization. Patients were encouraged for quadriceps strengthening exercises, ankle and calf exercises from day one and mobilized non-weight bearing from the second post-operative day depending upon pain tolerance of the patient. Surgical site suction drain was removed after $24 \mathrm{hr}$. The wound was inspected on the 3rd and 7th post-operative day. Stitches were remved after 14days. Patients followed up at 2 weeks, 4 weeks, 6 months and annually thereafter. Various parameters assessed on each follow up visit were:

Clinical: 1. Wound condition 2. Harris hip score

Radiological: 1. Union 2. Complication

Functional outcome was assessed with Harris Hip Scores on following parameters:

- Pain

- Limp

- Distance patient was able to walk with and without support 
- Use public transportation

- Stairs

- Use shoes and socks

- Absence of deformity

- Range of motion

Grading of Harris Hip Score:

$<70$ Points - Poor

70-79 Points - Fair

80-89 Points - Good

90-100 Points - Excellent

\section{Observations and Results}

The study involved 80 cases of unstable IT femur fracture of either sex followed up from June 2014 to June 2016. Out of 80 cases, 40 were treated by PFN (group A) and 40 were treated by DHS (group B). The results wereanalysed using students-t test and the two evaluated $p$ values were compared. In our study, maximum age was 80 years and minimum was 50 years. The average age was 64.3 years. In either group, 24 were OTA 31-A2 and 30 were $31-$ A3 type fractures. The Singh's index for osteoporosis showed that, both groups inclusive, there were 54 patients with grade 4 and above. In this study, Harris hip score at latest follow up was excellent in 23 patients $(46 \%)$ in group A and $20(36 \%)$ in group B, patients with good results were $15(28 \%)$ in group A and 20(45\%) in group B, patients with fair results were $4(6 \%)$ in group $A$ and $6(8 \%)$ in group B and patients poor results were $1(2 \%)$ in group B and no patient with poor results in group A.

Table 1: Harris Hip scores at latest follow up

\begin{tabular}{|l|c|c|}
\hline Harris Hip Score & PFN Group & DHS Group \\
\hline Excellent & $46 \%$ & $36 \%$ \\
\hline Good & $28 \%$ & $45 \%$ \\
\hline Fair & $6 \%$ & $8 \%$ \\
\hline Poor & Nil & $2 \%$ \\
\hline
\end{tabular}

In the D.H.S group, 1-month Harris hip score $($ mean $=23.5)$ was less than that of the P.F.N group (mean $=34.23)$ i.e. $\mathrm{p}<0.0001$. In 6 months, hip score in DHS (mean $=77.8$ ) was also less than that of PFN $($ mean $=81.8)$, i.e. $p$ value $=0.021$
Table 2: Comparative Hip scores at serial follow up

\begin{tabular}{|l|c|c|}
\hline Harris Hip Score & PFN Group & DHS Group \\
\hline 1-month follow up & 34.23 & 23.5 \\
\hline 6-months follow up & 81.8 & 77.8 \\
\hline
\end{tabular}

A comparison of time to union demonstrated no statistically significant differences between study groups $(\mathrm{P}=0.542)$. Out of $22 \mathrm{~A} 3$ fractures, in group $\mathrm{B}$ reduction loss occurred in 6 hips and in group A it was seen in 2 cases. Thus in unstable IT fractures reduction loss is significantly lower in group A than group B $(\mathrm{p}<0.0001)$.

The various parameters we had evaluated were as follows

- Intra operative blood loss as measured by mop count and collection in suction drain. The average blood loss in the PFN group was $105 \mathrm{ml}$ and in the DHS group was 270 $\mathrm{ml}$. Blood loss was less in PFN which is statistically significant, $\mathrm{p}$ value $<0.0005$.

- Intraoperative complications in PFN like failure to achieve close reduction in 3 patients where open reduction was performed. There was no iatrogenic fracture, difficulties in distal locking or any other intra-operative complication.

- Intraoperative complications in DHS: In 7 of the 40 cases there was improper placement of Richard's screw. Difficulties were encountered in reverse oblique fractures as the fracture site extended to entry point. There was varus angulation in 3 of 40 patients. On table surgeon had to switch to PFN in 2 cases in reverse oblique fracture. These cases were considered with PFN group for further follow up.

- Infection: There were 4 cases of superficial infection seen in the DHS group which were seen within 10 days of surgery and were treated by local debridement and antibiotics and did not require implant removal. No case of infection was seen in PFN group.

- Sliding: The sliding in both groups was compared at the end of 1 year on the $\mathrm{X}$ - 
rays as described by Hardy et al, there was an average of $5.50 \mathrm{~mm}$ of sliding in the P.F.N group as compared to $8.10 \mathrm{~mm}$ in the D.H.S group ( $\mathrm{p}<0.0005)$.

- Shortening: The average shortening in the PFN group was $5.35 \mathrm{~mm}$ as compared to $9.62 \mathrm{~mm}$ in the D.H.S group. So shortening is less in PFN group which is statistically significant. ( $\mathrm{p}$ value $<0.0005$ )

- Implant Failure: There was 4 of 50 case of implant failure in P.F.N group and revision surgery was required for two of those. The ' $Z$ ' pattern of implant failure was seen in both. In the D.H.S group there were 2 of 50 cases of implant failure, of which one was due to screw cut out and other was due to plate breakage. In both the cases revision surgery was required.

\section{Discussion}

The decision to use DHS or intramedullary nail is multifactorial and is based on surgeon training and preference, cost and patient and fracture characteristics. Proponents of intramedullary nail argue that less shortening occurs with an intramedullary nail than with a compression hip screw. In a recent study, minimal shortening (mean $5.9 \mathrm{~mm}$ ) was found at union in a series of inter-trochanteric femoral fractures considered "stable" and treated with DHS; similar shortening $(5.3 \mathrm{~mm})$ was found in "unstable" fractures treated with intramedullary nailing. The purpose was to show that experienced surgeons can identify stable intertrochanteric femoral fractures and that these can be treated with DHS with minimal shortening. Pajarinen et al. compared outcomes of PFN with DHS in the treatment of AO/OTA 31A fractures. At 4 months after surgery a much larger percentage of patients $(76 \%)$ treated with PFN returned to their pre-injury status than patients treated with DHS (54\%). The mean shortening of the femoral neck was also much less in patients treated with PFN $(1.3 \mathrm{~mm})$ than those treated with DHS (6.1mm).

Newer advances in intramedullary devices like InterTAN use two proximal inter-locking screws that allow linear intra-operative compression. The nail's geometry and integrated proximal interlocking at least theoretically improve stability in the proximal segment.

In our study we found:

- Less operative time in PFN group

- Less operative blood loss in PFN group

- Early return to daily activities in PFN group

- Less complication in PFN group as compared to DHS group.

In contrast, the plate and screw device will weaken the bone mechanically. The common causes of fixation failure are instability of the fractures, osteoporosis, and the lack of anatomical reduction, failure of fixation device and in-correct placement of screws.

\section{Conclusion}

Though PFN and DHS have similar outcomes in stable fractures, PFN has better functional outcome with unstable fractures. PFN requires shorter operation time and lesser soft tissue dissection; it has distinct advantages over DHS even in stable inter-trochanteric fractures. Hence from our study it may be concluded that, PFN is better fixation device for most unstable intertrochanteric fractures.

\section{References}

1. Melton L J, Kearns A E, Atkinson E J et al. Secular Trends in Hip Fracture Incidence and Recurrence, Osteoporosis International; 2009; 20(5): 687- 694.

2. Gullberg B, Johnell O, Kanis A. World Wide Projection for Hip Fracture, Osteoporosis International; 1997; 7(5):407-413.

3. Hardy D, Descamps P, Krallis P et al. Use of an Intramedullary Hip Screw Compared with a Compression Hip Screw with a Plate for Intertrochanteric Femoral Fractures. A Prospective Randomized Study of One Hundred Patients, The Journal of Bone \& Joint Surgery 1998; 80: 618-630 
4. Carter D, Hayes W. The Compressive Behavior of Bone as a Two-Phase Porous Structure, The Journal of Bone \& Joint Surgery 1977; 59A (7): 954-962.

5. Nuber $S$, Schoweiss $T$, Ruter $A$. Stabilization of unstable trochanteric femoral fractures: dynamic hip screw with trochanteric stabilization plate vs Proximal femoral nail, Journal of orthopaedic trauma 2003; 17(4): 316-317.

6. Steinberg E L, Nehemia N H, Shmuel D L et al. The proximal femur nailing system: biomechanical properties of the nail and a cadaveric study, Journal of Biomechanics; 2005; 38: 63-68.

7. M Faisal, $P$ Nistane. Proximal Femoral Nailing vs. Dynamic Hip Screw in unstable IntertrochantericFracture of Femur - A comparative analysis, International Journal of Biomedical and Advance Research

8. Rockwood and Green's Fractures in Adults Eighth Edition, Wolters Kluwer

9. Campbell's Operative Orthopaedics, Twelfth edition, Elsevier Publishers 